\title{
ON THE HEAT KERNEL OF THE BERGMANN METRIC ON ALGEBRAIC VARIETIES
}

\author{
PETER LI AND GANG TIAN
}

\section{INTRODUCTION}

Let $M$ be an $n$-dimensional algebraic variety in a projective space $\mathbb{C P}^{n+l}$ of dimension $n+l$ with $l>0$. If we denote the singular set of $M$ by $\Sigma_{M}$, then the restriction of the standard Fubini-Study metric of $\mathbb{C P}^{n+l}$ to $M \backslash \Sigma_{M}$ is an incomplete Kähler metric, $g$, called the Bergmann metric. Let us consider the operators $d$ and $\delta$ defined on $C^{1}$ functions and $C^{1} 1$-forms on $M \backslash \Sigma_{M}$, respectively. We define the domain $\mathscr{D}(d)$ of $d$ to be the set of $C^{1}$ functions $f$ defined on $M \backslash \Sigma_{M}$ such that both $f$ and $d f$ are in $L^{2}$. Similarly, we define the domain $\mathscr{D}(\delta)$ of $\delta$ to be the set of $C^{1} 1$-forms $\omega$ such that both $\omega$ and $\delta \omega$ are in $L^{2}$. We then define the Laplacian $\Delta$ with respect to the Bergmann metric by $\Delta=-\delta d$ with domain $\mathscr{D}(\Delta)$ given by the set of $C^{2}$ functions $f$ such that $f \in \mathscr{D}(d)$ and $d f \in \mathscr{D}(\delta)$. The main purpose of this paper is to show that:

Main Result. The Laplacian for functions $\Delta$ is essentially self-adjoint on $M \backslash \Sigma_{M}$. The heat semi-group $e^{\Delta t}$ generated by $\Delta$ has a kernel $H(x, y, t)$ in $\mathscr{D}(\Delta)$ as a function of $x$ or $y$. Also, $H(x, y, t)$ is symmetric in the variables $x$ and $y$, and it satisfies the conservation property

$$
\int_{M \backslash \Sigma_{M}} H(x, y, t) d y=1
$$

for all $x \in M \backslash \Sigma_{M}$. If we denote $\tilde{H}(r(\tilde{x}, \tilde{y}), t)=H(\tilde{x}, \tilde{y}, t)$ to be the rotationally symmetric heat kernel on the standard $\mathbb{C P}^{n}$, then for $x, y \in M \backslash \Sigma_{M}$ and $t>0$, we have

$$
H(x, y, t) \leq \tilde{H}\left(r_{x}(y), t\right)
$$

Moreover, equality holds if and only if $M$ is a totally geodesic $\mathbb{C P}^{n}$.

As an interesting corollary of this upper bound, we derive a universal lower bound for the $d^{\text {th }}$ non-zero eigenvalue for algebraic varieties of degree $d$.

Received by the editors August 17, 1993 and, in revised form, May 19, 1994.

1991 Mathematics Subject Classification. Primary 58C40, 58F19, 58G11, 58G25.

Key words and phrases. Algebriac variety, heat kernel, Laplacian.

The first author's research was supported in part by NSF grant \#DMS-93004225. The second author's research was supported in part by a Sloan Fellowship and NSF grant \#DMS-9303999. 
Corollary 5.5. Let $M^{n}$ be an algebraic variety of degree $d$. There exists a constant $C(n, d)>0$ depending only on $n$ and $d$ alone, such that the $d^{\text {th }}$ non-zero eigenvalue with respect to the Bergmann metric on $M$ satisfies

$$
\lambda_{d} \geq C(n, d) .
$$

The first two sections of this paper are devoted to proving the upper bound of $H(x, y, t)$ for the case when $M$ is an algebraic submanifold. In fact, the argument is valid even if $M$ has boundary, and the heat kernel is taken to be the Dirichlet heat kernel. The idea is to transplant the heat kernel $\tilde{H}$ for $\mathbb{C P}^{n}$ onto $M$ via the extrinsic distance function $r_{x}(y)$ given by the Fubini-Study metric of $\mathbb{C P}^{n+l}$. This argument originated in a previous work of Cheng-LiYau [C-L-Y2], where they developed comparison theorems for the heat kernels of minimal submanifolds in $\mathbb{R}^{n+l}, \mathbb{H}^{n+l}$, and $\mathbb{S}^{n+l}$.

In $\S 3$, we consider the case when $M$ is a singular variety in $\mathbb{C P}^{n+l}$ with singular set $\Sigma_{M}$. We will show that if $H_{\epsilon}$ is the heat kernel for the Dirichlet Laplacian on the manifold $M_{\epsilon}$ with boundary obtained by excising the $\epsilon$-tubular neighborhood of the singular set, then $H_{\epsilon}$ converges uniformly on compact subsets of $M \backslash \Sigma_{M}$ to a heat kernel $H$ as $\epsilon \rightarrow 0$. Using the upper bound of $H_{\epsilon}$ and by passing through the limit, we conclude that the upper bound also holds for $H$. The conservation property of $H$ will be established as a consequence. Furthermore, if we denote $K_{\epsilon}$ to be the Neumann heat kernel on $M_{\epsilon}$, then we will show that $K_{\epsilon}$ also converges to $H$ uniformly on compact subsets of $M \backslash \Sigma_{M}$.

As a separate issue, we will prove in $\S 4$ that the Laplacian $\Delta$ on $M$ has a self-adjoint closure. Hence the heat kernel that one can obtain using semigroup theory coincides with the one constructed in $\S 3$. Let us point out that for normal singular algebraic surfaces with isolated point singularities, the selfadjointness of $\Delta$ was proved by Nagase [N]. Recently, Pati [P2] proved the self-adjointness of $\Delta$ for algebraic threefolds with isolated singularities. Our method here applies to any singular manifold (not necessarily algebraic) with real codimension 2 singularities.

In the last section, we will show that the operator $\Delta-1$ is invertible in $L^{2}(M)$ and its inverse $(\Delta-1)^{-1}$ is a compact operator. In particular, this shows that $\Delta$ has discrete spectrum. The upper bound on $H$ implies that the heat operator is of trace class and an upper bound for the asymptotic behavior of $H$ follows (Corollary 5.4) as a consequence. We would like to point out that a weaker estimate of the type similar to Corollary 5.4 was obtained by Nagase [N] for algebraic surfaces with isolated singularities and by Pati [P1] for algebraic threefolds with isolated singularities. Finally, we will also prove Corollary 5.5 in the last section.

The authors would like to thank the referee for the valuable comments. The thoroughness of the referee report helped us in presenting the paper in a more readable form.

\section{THe HEAT KeRNel OF $\mathbb{C P}^{n}$}

In this section, we will derive some properties for the heat kernel $\tilde{H}$ of the model space $\mathbb{C P}^{n}$. Let $x \in \mathbb{C P}^{n}$ be a fixed point of $\mathbb{C P}^{n}$. The geodesic distance 
from any point $y \in \mathbb{C P}^{n}$ to the point $x$ is denoted by $r_{x}(y)$. When ambiguity does not arise, we will simply write $r(y)=r_{x}(y)$. By the symmetry of $\mathbb{C P}^{n}$ and the uniqueness of the heat kernel, we can express the heat kernel $\tilde{H}\left(r_{x}(y), t\right)$ of $\mathbb{C P}^{n}$ as a function of the distance and time.

To unify our point of view for the sake of future reference, we will consider the Fubini-Study metric of $\mathbb{C P}^{n}$ by thinking of it as an embedded submanifold in $\mathbb{C P}^{n+l}$. To that end, for a fixed point $x \in \mathbb{C P}^{n+l}$, we can choose coordinates $z=\left(z_{1}, z_{2}, \ldots, z_{n+l}\right)$ of $\mathbb{C P}^{n+l}$ on $\mathbb{C}^{n+l}$ with $x=(0,0, \ldots, 0)$. Using this coordinate system, one can express the Fubini-Study metric by $\partial \check{\partial} \log \left(1+|z|^{2}\right)$, with $|z|^{2}=\sum_{i=1}^{n+l}\left|z_{i}\right|^{2}$. Then the distance function to $x$ is simply given by $r_{x}(z)=\arctan |z|$. We observe that the cut-locus of $x$ is a copy of $\mathbb{C P}^{n+l-1}$ given by $\left\{z \in \mathbb{C P}^{n+l} \mid r_{x}(z)=\frac{\pi}{2}\right\}$. If we define the increasing function

$$
s(r)=\log \left(1+\tan ^{2} r\right)=-2 \log (\cos r)
$$

for $r \in\left[0, \frac{\pi}{2}\right)$, then the Fubini-Study metric can be written as $\partial \bar{\partial} s(r(z))$. In general, if $M$ is an algebraic submanifold of dimension $n$ in $\mathbb{C P}^{n+l}$, the Bergmann metric is given by restricting $g_{i j} d z_{i} \wedge d z_{\bar{j}}=\partial \bar{\partial} s(r(z))$ to $M$. In terms of these coordinates, the Laplacian of the Bergmann metric on $M$ can be written as $\tilde{\Delta}=4 g^{i j} \frac{\partial^{2}}{\partial z_{i} \partial z_{j}}$, hence clearly $\tilde{\Delta} s=4 n$. In this setting, $\mathbb{C P}^{n}$ can be thought of as the submanifold given by $\left\{z \in \mathbb{C P}^{n+l} \mid z=\left(z_{1}, \ldots, z_{n}, 0, \ldots, 0\right)\right\}$. Writing the heat kernel of $\mathbb{C P}^{n}$ as a function of $s$ and $t$, we can express the heat equation as

$$
\begin{aligned}
\left(\tilde{\Delta}_{y}-\frac{\partial}{\partial t}\right) \tilde{H}(x, y, t) & =\left(\tilde{\Delta}_{y}-\frac{\partial}{\partial t}\right) \tilde{H}\left(s\left(r_{x}(y)\right), t\right) \\
& =\tilde{H}_{s} \tilde{\Delta}_{s}+\tilde{H}_{s s}|\tilde{\nabla} s|^{2}-\tilde{H}_{t} \\
& =4 n \tilde{H}_{s}+4\left(e^{s}-1\right)\left|\tilde{\nabla}^{2}\right|^{2} \tilde{H}_{s s}-\tilde{H}_{t} \\
& =4 n \tilde{H}_{s}+4\left(e^{s}-1\right) \tilde{H}_{s s}-\tilde{H}_{t}
\end{aligned}
$$

where $\tilde{\nabla}$ and $\tilde{\Delta}$ are the gradient and the Laplacian with respect to the FubiniStudy metric on $\mathbb{C P}^{n}$.

Proposition 1.1. The heat kernel of $\mathbb{C P}^{n}$ with respect to the Fubini-Study metric satisfies the differential inequality

$$
4\left(e^{s}-1\right) \tilde{H}_{s s}(s, t) \geq 0
$$

for all $(s, t) \in[0, \infty) \times[0, \infty)$.

Proof. Let us denote $\psi(s, t)=4\left(e^{s}-1\right) \tilde{H}_{s s}$. We will first prove that

$$
\lim _{s \rightarrow 0} \psi(s, t)=0
$$

for all $t>0$. Since

$$
\tilde{\Delta} \tilde{H}=4 n \tilde{H}_{s}+4\left(e^{s}-1\right) \tilde{H}_{s s},
$$


we can write

$$
\psi=\tilde{\Delta} \tilde{H}-\frac{2 n}{\tan r} \tilde{H}^{\prime}
$$

with $\tilde{H}^{\prime}=\frac{\partial \tilde{H}}{\partial r}$. Observe that because $\tilde{H}$ is rotationally symmetric, $\tilde{H}^{\prime}(0, t)$ must vanish for all $t>0$. Hence by l'Hopital's rule and the fact that $s=$ $-2 \log (\cos r)$,

$$
\begin{aligned}
\lim _{s \rightarrow 0} \psi(s, t) & =\lim _{r \rightarrow 0} \psi(s(r), t) \\
& =\tilde{\Delta} \tilde{H}(0, t)-2 n \tilde{H}^{\prime \prime}(0, t) .
\end{aligned}
$$

However, direct computation shows that

$$
\tilde{\Delta} \tilde{H}(s(r), t)=\tilde{H}^{\prime \prime}(r, t)+\frac{2 n-1}{\tan r} \tilde{H}^{\prime}(r, t)-\tan r \tilde{H}^{\prime}(r, t) .
$$

Hence by taking $r \rightarrow 0$ we have

$$
\begin{aligned}
\tilde{\Delta} \tilde{H}(0, t) & =\tilde{H}^{\prime \prime}(0, t)+(2 n-1) \tilde{H}^{\prime \prime}(0, t) \\
& =2 n \tilde{H}^{\prime \prime}(0, t) .
\end{aligned}
$$

This verifies the claim that $\lim _{s \rightarrow 0} \psi(s, t)=0$ for all $t>0$.

Note that since $\tilde{H}(s, 0)=0$ for all $s \in(0, \infty), \psi(s, 0)=0$ for $s \in(0, \infty)$. We now claim that $\psi(s, t) \geq 0$ for $(s, t)$ sufficiently close to $(0,0)$. To see this, we consider the eigenfunction expansion of $\tilde{H}(s, t)$.

Let $\left\{0=\lambda_{0}<\lambda_{1} \leq \cdots \leq \lambda_{i} \leq \cdots\right\}$ be the set of discrete eigenvalues for the Laplacian on $\mathbb{C P}^{n}$ with associated eigenfunctions $\phi_{i}$ satisfying

$$
\tilde{\Delta} \phi_{i}=-\lambda_{i} \phi_{i}
$$

The heat kernel can be written as

$$
\tilde{H}(x, y, t)=\sum_{i=0}^{\infty} e^{-\lambda_{i} t} \phi_{i}(x) \phi_{i}(y) .
$$

Using the argument of Lemma 7 in [C-L-Y2], we claim that for any fixed point $x \in \mathbb{C P}^{n}$ and for each eigenvalue $\lambda$, there exists a unique eigenfunction $\phi_{\lambda}$ in that eigenspace $E_{\lambda}$ satisfying $\phi_{\lambda}(x)>0$ with $\left\|\phi_{\lambda}\right\|_{L^{2}}=1$ and $\phi_{\lambda}(y)=$ $\phi_{\lambda}\left(r_{x}(y)\right)$ is rotationally symmetric about the point $x$. Moreover, the subspace perpendicular to $\phi_{\lambda}$ with respect to the $L^{2}$ inner product is given by the set of functions $E_{\lambda}^{0}=\left\{f \in E_{\lambda} \mid f(x)=0\right\}$. For the sake of completeness, let us outline the argument here. Let us first observe that the codimension of $E_{\lambda}^{0}$ in $E_{\lambda}$ is at most one. Let $f$ and $h$ be two eigenfunctions in $E_{\lambda}$ which are perpendicular to $E_{\lambda}^{0}$. Clearly, we can find constants $a, b$ such that $a f(x)+b h(x)=0$. This implies that the function $a f+b h$ is in $E_{\lambda}^{0}$. This is impossible unless $a f+b h \equiv 0$. Hence the span of $\{f, h\}$ is 1 -dimensional. We now claim that $E_{\lambda}^{0} \neq E_{\lambda}$. Otherwise, all eigenfunctions in $E_{\lambda}$ will satisfy the condition $f(x)=0$. In particular, if $\left\{f_{1}, \ldots, f_{k}\right\}$ is an orthonormal basis for $E_{\lambda}$, then the function $F=\sum_{i=1}^{k} f_{i}^{2}$ satisfies $F(x)=0$. On the other hand, because $\mathbb{C P}^{n}$ 
is a homogeneous manifold, $F$ is a constant function by [L]. This implies that $F \equiv 0$ which is a contradiction. Let $\phi_{\lambda}$ be an eigenfunction in the perpendicular subspace of $E_{\lambda}^{0}$ in $E_{\lambda}$ which has unit $L^{2}$-norm. Clearly, $\phi_{\lambda}(x) \neq 0$ and we may choose $\phi_{\lambda}(x)>0$. These two conditions uniquely determine $\phi_{\lambda}$. Since the isotropic subgroup of isometries at $x$ acts transitively on the unit tangent sphere, this implies that $\phi_{\lambda}$ is rotational symmetric.

Using this, we can write

$$
\tilde{H}\left(r_{x}(y), t\right)=\sum_{\lambda} e^{-\lambda t} \phi_{\lambda}(0) \phi_{\lambda}\left(r_{x}(y)\right) .
$$

Equation (1.2) asserts that the function $\psi(s(r), t)$ can be expressed as

$$
\psi(r, t)=\sum_{\lambda} e^{-\lambda t} \phi_{\lambda}(0)\left(-\lambda \phi_{\lambda}-\frac{2 n}{\tan r} \phi_{\lambda}^{\prime}\right) .
$$

Clearly, by the fact that $\phi_{\lambda}>0$, we only need to show that

$$
\lambda \phi_{\lambda}+\frac{2 n}{\tan r} \phi_{\lambda}^{\prime}<0
$$

for all $\lambda$. From now on, let us suppress the index $\lambda$ and define

$$
g=\lambda \phi+2 n \frac{\phi^{\prime}}{\tan r} .
$$

We will show that $g(0)=g^{\prime}(0)=0$ and that

$$
g^{\prime \prime}(0)=\frac{-\lambda^{2}}{2 n(n+1)} \phi(0) \text {. }
$$

This is sufficient to show our claim that $\psi \geq 0$ near $(r, t)=(0,0)$.

Note that the rotational symmetry of $\phi$ implies that $\phi^{\prime}(0)=0$. Letting $r \rightarrow 0$ and using l'Hopital's rule,

$$
\begin{aligned}
\lim _{r \rightarrow 0} g(r) & =\lambda \phi(0)+2 n \lim _{r \rightarrow 0} \frac{\phi^{\prime}(r)}{\tan r} \\
& =\lambda \phi(0)+2 n \lim _{r \rightarrow 0} \frac{\phi^{\prime \prime}(r)}{\left(1+\tan ^{2} r\right)} \\
& =\lambda \phi(0)+2 n \phi^{\prime \prime}(0) .
\end{aligned}
$$

On the other hand,

$$
\begin{aligned}
-\lambda \phi & =\tilde{\Delta} \phi \\
& =\phi^{\prime \prime}+\frac{2 n-1}{\tan r} \phi^{\prime}-\tan r \phi^{\prime}
\end{aligned}
$$

implies that

$$
\begin{aligned}
\phi^{\prime \prime}(0) & =-\lambda \phi(0)-(2 n-1) \lim _{r \rightarrow 0} \frac{\phi^{\prime}(r)}{\tan r} \\
& =-\lambda \phi(0)-(2 n-1) \phi^{\prime \prime}(0) .
\end{aligned}
$$

Hence $2 n \phi^{\prime \prime}(0)=-\lambda \phi(0)$ and $g(0)=0$. 
Directly differentiating (1.4) and substituting (1.5) gives

$$
\begin{aligned}
g^{\prime}(r) & =\lambda \phi^{\prime}(r)+2 n \frac{\phi^{\prime \prime}(r)}{\tan r}-2 n \frac{\phi^{\prime}(r)}{\tan ^{2} r}-2 n \phi^{\prime}(r) \\
& =\lambda \phi^{\prime}(r)-2 n \frac{g(r)}{\tan r} .
\end{aligned}
$$

Hence

$$
\begin{aligned}
g^{\prime}(0) & =-2 n \lim _{r \rightarrow 0} \frac{g(r)}{\tan r} \\
& =-2 n \lim _{r \rightarrow 0} \frac{g^{\prime}(r)}{\left(1+\tan ^{2} r\right)} \\
& =-2 n g^{\prime}(0)
\end{aligned}
$$

and $g^{\prime}(0)=0$. Differentiating (1.6) yields

$$
\begin{aligned}
g^{\prime \prime}(r) & =\lambda \phi^{\prime \prime}(r)-2 n \frac{g^{\prime}(r)}{\tan r}+2 n \frac{g(r)}{\tan ^{2} r}+2 n g(r) \\
& =-\lambda^{2} \phi-\lambda(2 n-1) \frac{\phi^{\prime}(r)}{\tan r}+\lambda \tan r \phi^{\prime}(r)-2 n \frac{g^{\prime}(r)}{\tan r}+2 n \frac{g(r)}{\tan ^{2} r}+2 n g(r) \\
& =(2 n-\lambda) g(r)+\lambda \frac{\phi^{\prime}(r)}{\tan r}+\lambda \tan r \phi^{\prime}(r)-2 n \frac{g^{\prime}(r)}{\tan r}+2 n \frac{g(r)}{\tan ^{2} r} .
\end{aligned}
$$

Therefore

$$
\begin{aligned}
g^{\prime \prime}(0) & =\lambda \phi^{\prime \prime}(0)-2 n g^{\prime \prime}(0)+2 n \lim _{r \rightarrow 0} \frac{g^{\prime}(r)}{2 \tan r\left(1+\tan ^{2} r\right)} \\
& =\frac{-\lambda^{2}}{2 n} \phi(0)-2 n g^{\prime \prime}(0)+2 n \lim _{r \rightarrow 0} \frac{g^{\prime \prime}(r)}{2\left(1+\tan ^{2} r\right)+6 \tan ^{2} r\left(1+\tan ^{2}\right)} \\
& =\frac{-\lambda^{2}}{2 n} \phi(0)-n g^{\prime \prime}(0)
\end{aligned}
$$

and

$$
g^{\prime \prime}(0)=\frac{-\lambda^{2}}{2 n(n+1)} \phi(0)
$$

This proves our claim that $\psi(s(r), t) \geq 0$ near $(r, t)=(0,0)$.

Note that by (1.2), (1.3), and that $s \rightarrow \infty$ is equivalent to $r \rightarrow \frac{\pi}{2}$, we have

$$
\begin{aligned}
\lim _{s \rightarrow \infty} \psi(s, t) & =\lim _{r \rightarrow \frac{\pi}{2}}\left(\tilde{\Delta} \tilde{H}(r, t)-\frac{2 n}{\tan r} \tilde{H}^{\prime}(r, t)\right) \\
& =\lim _{r \rightarrow \frac{\pi}{2}}\left(\tilde{H}^{\prime \prime}(r, t)-\frac{1}{\tan r} \tilde{H}^{\prime}(r, t)-\tan r \tilde{H}^{\prime}(r, t)\right) .
\end{aligned}
$$

The fact that $\tilde{H}$ is rotationally symmetric and that the set $r=\frac{\pi}{2}$ constitutes 
the cut-locus imply that $\tilde{H}^{\prime}\left(\frac{\pi}{2}, t\right)=0$. Therefore (1.7) becomes

$$
\begin{aligned}
\lim _{s \rightarrow \infty} \psi(s, t) & =\lim _{r \rightarrow \frac{\pi}{2}} \tilde{H}^{\prime \prime}(r, t)-\lim _{r \rightarrow \frac{\pi}{2}} \frac{1}{\cos r}\left(\sin r \tilde{H}^{\prime}(r, t)\right) \\
& =\lim _{r \rightarrow \frac{\pi}{2}} \tilde{H}^{\prime \prime}(r, t)+\lim _{r \rightarrow \frac{\pi}{2}} \frac{1}{\sin r}\left(\sin r \tilde{H}^{\prime \prime}(r, t)+\cos r \tilde{H}^{\prime}(r, t)\right) \\
& =2 \lim _{r \rightarrow \frac{\pi}{2}} \tilde{H}^{\prime \prime}(r, t) .
\end{aligned}
$$

However, $\mathbb{C P}^{n}$ is a Ricci model in the sense of Cheeger-Yau [C-Y], and they proved that $\tilde{H}^{\prime}(r, t) \leq 0$ for all $0 \leq r \leq \frac{\pi}{2}$ and for all $t>0$. For the sake of completeness, we will give an outline of the argument here. Since

$$
\tilde{H}^{\prime}=2 \tilde{H}_{s} \tan r
$$

it suffices to show that $\tilde{H}_{s} \leq 0$. At the point $r=0$, we see that

$$
\begin{aligned}
\lim _{r \rightarrow 0} 2 \tilde{H}_{s}(s(r), t) & =\lim _{r \rightarrow 0^{-}} \frac{\tilde{H}^{\prime}(r, t)}{\tan r} \\
& =\tilde{H}^{\prime \prime}(0, t) .
\end{aligned}
$$

However, by (1.3)

$$
\begin{aligned}
\frac{\partial}{\partial t} \tilde{H}(0, t) & =\tilde{\Delta} \tilde{H}(0, t) \\
& =\tilde{H}^{\prime \prime}(0, t)+(2 n-1) \lim _{r \rightarrow 0} \frac{\tilde{H}^{\prime}(r, t)}{\tan r} \\
& =2 n \tilde{H}^{\prime \prime}(0, t) .
\end{aligned}
$$

Also using the semigroup property, we deduce that

$$
\frac{\partial}{\partial t} \tilde{H}(0, t)=-2 \int|\tilde{\nabla} \tilde{H}|^{2}\left(r(y), \frac{t}{2}\right) d y .
$$

Hence $\tilde{H}^{\prime \prime}(0, t) \leq 0$ and $\lim _{r \rightarrow 0} 2 \tilde{H}_{s}(s(r), t) \leq 0$. When $r=\frac{\pi}{2}$, using the fact that $\tilde{H}^{\prime}\left(\frac{\pi}{2}, t\right)=0$, we see that $\lim _{r \rightarrow \frac{\pi}{2}} \tilde{H}_{s}(s(r), t)=0$. We can now conclude that $\tilde{H}_{s}(s(r), t) \leq 0$ by using the maximum principle because $\tilde{H}_{s}$ satisfies the equation

$$
\frac{\partial}{\partial t} \tilde{H}_{s}=4\left(e^{s}-1\right) \tilde{H}_{s s s}+\left(n+4 e^{s}\right) \tilde{H}_{s s}
$$

which can be obtained by differentiating the heat equation (1.1) with respect to $s$. Using this and the fact that $\tilde{H}^{\prime}\left(\frac{\pi}{2}, t\right)=0$ we conclude that $\tilde{H}^{\prime \prime}\left(\frac{\pi}{2}, t\right) \geq 0$. Hence the function $\psi(s, t)$ satisfies the boundary condition that $\psi(s, t) \geq 0$ on $\{(s, t) \mid(0 \times[0, \infty)) \cup([0, \infty) \times 0) \cup(\infty \times[0, \infty))\}$.

On the other hand, differentiating (1.1) with respect to $s$ twice yields

$$
\psi_{s s}=\tilde{H}_{s s t}-n \tilde{H}_{s s s} .
$$

The definition of $\psi$ implies that

$$
\psi_{s}=4\left(e^{s}-1\right) \tilde{H}_{s s}+4 e^{s} \tilde{H}_{s s},
$$


and hence

$$
4\left(e^{s}-1\right) \psi_{s s}+n \psi_{s}-\frac{4 e^{s}}{e^{s}-1} \psi-\psi_{t}=0
$$

Applying the minimum principle to this parabolic equation, we conclude that $\psi \geq 0$ on $[0, \infty) \times[0, \infty)$.

\section{UPPER BOUND OF THE HEAT KERNEL FOR ALGEBRAIC SUBMANIFOLDS}

We are now ready to prove an upper bound for the heat kernel on an algebraic submanifold of $\mathbb{C} \mathbb{P}^{n+l}$.

Theorem 2.1. Let $M^{n}$ be an $n$-dimensional algebraic submanifold of $\mathbb{C P}^{n+l}$. For a fixed point $x \in M$, let us denote the distance function of $\mathbb{C P}^{n+l}$ when restricted to $M$ by $r_{x}(y)$. Suppose $H(x, y, t)$ is the heat kernel of $M$ with respect to the Bergmann metric. When $\partial M \neq \varnothing, H(x, y, t)$ is taken to be the heat kernel with Dirichlet boundary condition. For all $x, y \in M$ and $t \in[0, \infty)$, we have

$$
H(x, y, t) \leq \tilde{H}\left(r_{x}(y), t\right)
$$

where $\tilde{H}\left(r_{x}(y), t\right)$ is the transplanted heat kernel of $\mathbb{C P}^{n}$. Moreover, if $M$ has empty boundary and

$$
H(x, y, t)=\tilde{H}\left(r_{x}(y), t\right),
$$

then $M$ must be a totally geodesic $\mathbb{C P}^{n}$ in $\mathbb{C P}^{n+l}$.

Proof. We will first show that the transplanted heat kernel $\tilde{H}\left(r_{x}(y), t\right)$ satisfies the differential inequality

$$
\left(\Delta_{y}-\frac{\partial}{\partial t}\right) \tilde{H}\left(r_{x}(y), t\right) \leq 0
$$

for $x, y \in M$ such that $r_{x}(y)<\frac{\pi}{2}$, and for $t \in(0, \infty)$. To see this, we will express the heat kernel in the variable $s(r)=-2 \log (\cos r)$ as in $\S 1$. Formula (1.1), Proposition 1.1, and the fact that $\left|\nabla_{y} r_{x}(y)\right|^{2} \leq 1$ imply that

$$
\begin{aligned}
\left(\Delta_{y}-\frac{\partial}{\partial t}\right) \tilde{H}\left(s\left(r_{x}(y)\right), t\right) & =4 n \tilde{H}_{s}+4\left(e^{s}-1\right)|\nabla r|^{2} \tilde{H}_{s s}-\tilde{H}_{t} \\
& \leq 4 n \tilde{H}_{s}+4\left(e^{s}-1\right) \tilde{H}_{s s}-\tilde{H}_{t} \\
& =\left(\tilde{\Delta}-\frac{\partial}{\partial t}\right) \tilde{H}\left(s\left(r_{x}(y)\right), t\right) \\
& =0 .
\end{aligned}
$$

Hence (2.1) is justified. We would like to point out that the above argument only requires the inequality $\Delta s \geq 4 n$.

To prove the inequality between $H$ and $\tilde{H}$, we first observe that for $x, y \in$ $M$ the transplanted kernel satisfies $\lim _{t \rightarrow 0} \tilde{H}\left(r_{x}(y), t\right)=\delta_{x}(y)$. Let us also point out that the transplanted function $\tilde{H}\left(r_{x}(y), t\right)$ can be defined on $\mathbb{C P}^{n+l}$. In fact, for any pair of points $x, y \in \mathbb{C P}^{n+l}$, there is a totally geodesic $\mathbb{C P}^{n}$ passing through them, hence the distance function $r_{x}(y)$ of $\mathbb{C P}^{n+l}$ can be viewed as the distance function of the totally geodesic $\mathbb{C P}^{n}$. The transplanted function 
$\tilde{H}\left(r_{x}(y), t\right)$ defined this way will be a smooth function on $\mathbb{C P}^{n+l}$ since the heat kernel $\tilde{H}$ is smooth on $\mathbb{C P}^{n}$. The restriction of $\tilde{H}$ on $M$ is then a smooth transplanted kernel. By Duhamel's principle and (2.1), we can write

$$
\begin{gathered}
\tilde{H}\left(r_{x}(y), t\right)-H(x, y, t) \\
=\int_{0}^{t} \frac{\partial}{\partial s}\left\{\int_{M} \tilde{H}\left(r_{x}(z), s\right) H(z, y, t-s) d z\right\} d s \\
=\int_{0}^{t} \int_{M} \frac{\partial \tilde{H}}{\partial s}\left(r_{x}(z), s\right) H(z, y, t-s) d z d s \\
\quad-\int_{0}^{t} \int_{M} \tilde{H}\left(r_{x}(z), s\right) \frac{\partial H}{\partial(t-s)}(z, y, t-s) d z d s \\
\geq \int_{0}^{t} \int_{M} \Delta_{z} \tilde{H}\left(r_{x}(z), s\right) H(z, y, t-s) d z d s \\
\quad-\int_{0}^{t} \int_{M} \tilde{H}\left(r_{x}(z), s\right) \Delta_{z} H(z, y, t-s) d z d s \\
=\int_{0}^{t} \int_{\partial M} \frac{\partial \tilde{H}}{\partial \nu_{z}}\left(r_{x}(z), s\right) H(z, y, t-s) d z d s \\
\quad-\int_{0}^{t} \int_{\partial M} \tilde{H}\left(r_{x}(z), s\right) \frac{\partial H}{\partial \nu_{z}}(z, y, t-s) d z d s
\end{gathered}
$$

for $x, y \in M$ and $t>0$. Using the fact that $H$ is positive in the interior of $M$ and that it satisfies the Dirichlet boundary condition on $\partial M$, we observe that $\frac{\partial H}{\partial \nu_{z}}(z, y, t-s)<0$ for $z \in \partial M$. Together with the fact that $\tilde{H}$ is positive, we conclude that

$$
\tilde{H}\left(r_{x}(y), t\right)-H(x, y, t) \geq 0 .
$$

When $M$ has empty boundary and equality occurs, it follows that

$$
\left|\nabla r_{x}(z)\right|=1
$$

for all $z \in M$. If we choose coordinates for $\mathbb{C P}^{n+l}$ around $x$ so that $M$ is tangent to the totally geodesic $\mathbb{C P}^{n}$ given by $\left\{z \mid\left(z_{1}, \ldots, z_{n}, 0, \ldots, 0\right)\right\}$, then (2.2) together with the fact that $r_{x}(z)$ is the distance function of $\mathbb{C P}^{n+l}$ implies that $M=\mathbb{C P}^{n}$.

\section{HEAT KERNEL FOR SINGULAR VARIETIES}

Let us first remark that since the singular set of an algebraic variety is of at least real codimension 2, we can construct a heat kernel on $M$ by using the Dirichlet heat kernels on the complement of tubular neighborhoods of the singular set. More precisely, let us denote $\Sigma_{M}$ to be the singular set of $M$ and $T_{\epsilon}=\left\{x \in M \mid r\left(x, \Sigma_{M}\right) \leq \epsilon\right\}$ to be the intrinsic (or extrinsic) $\epsilon$-neighborhood of $\Sigma_{M}$. Define $M_{\epsilon}=M \backslash T_{\epsilon}$ and let $H_{\epsilon}(x, y, t)$ be the Dirichlet heat kernel on $M_{\epsilon}$. The maximum principle asserts that $H_{\epsilon}(x, y, t) \geq H_{\epsilon^{\prime}}(x, y, t)$ for all $x, y \in M_{\epsilon^{\prime}}$ and $t>0$, if $\epsilon<\epsilon^{\prime}$. On the other hand, Theorem 2.1 implies that $H_{\epsilon}(x, y, t)$ is uniformly bounded from above for any fixed $t>0$. Hence 
using the local gradient estimate of $H_{\epsilon}$ by [L-Y], we conclude that there exists a function $H(x, y, t)$ such that $H_{\epsilon}(x, y, t) \nearrow H(x, y, t)$ as $\epsilon \rightarrow 0$ on compact subsets of $\left(M \backslash \Sigma_{M}\right) \times\left(M \backslash \Sigma_{M}\right) \times(0, \infty)$. In fact, $H(x, y, t)$ is smooth on $\left(M \backslash \Sigma_{M}\right) \times\left(M \backslash \Sigma_{M}\right) \times(0, \infty)$ and it is a heat kernel on $M$. Clearly, by passing the estimate of Theorem 2.1 through the limit,

$$
H(x, y, t) \leq \tilde{H}\left(r_{x}(y), t\right) .
$$

In particular, $H(x, \cdot, t) \in L^{2}(M)$.

Lemma 3.1. The heat kernel constructed above is in $H_{1,2}(M)$ as a function of either the $x$ or the $y$ variable. Moreover, it satisfies

$$
\int_{M} H(x, y, t) d y=1
$$

for all $x \in M$ and $t>0$. Hence it is the unique heat kernel on $M$ satisfying these properties.

Proof. The proof of Lemma 7 in [C-L-Y1] asserts that

$$
\int_{M_{\epsilon}}\left|\Delta H_{\epsilon}\right|^{2}(x, y, t) d y \leq C t^{-2} \int_{M_{\epsilon}} H_{\epsilon}^{2}\left(x, y, \frac{t}{2}\right) d y .
$$

However, monotonicity of $H_{\epsilon}$ implies that

$$
\int_{M_{\epsilon}} H_{\epsilon}^{2}\left(x, y, \frac{t}{2}\right) d y \leq \int_{M_{\epsilon}} H^{2}\left(x, y, \frac{t}{2}\right) d y \leq \int_{M} H^{2}\left(x, y, \frac{t}{2}\right) d y .
$$

If we let $0<\epsilon<\epsilon_{0}$, then the above inequalities imply that

$$
\int_{M_{\epsilon_{0}}}\left|\Delta H_{\epsilon}\right|^{2}(x, y, t) d y \leq C t^{-2} \int_{M} H^{2}\left(x, y, \frac{t}{2}\right) d y .
$$

Letting $\epsilon \rightarrow 0$ and using the fact that $H_{\epsilon} \rightarrow H$ uniformly on compact subsets of $\left(M \backslash \Sigma_{M}\right) \times\left(M \backslash \Sigma_{M}\right) \times(0, \infty)$ yields

$$
\int_{M_{\epsilon_{0}}}|\Delta H|^{2}(x, y, t) d y \leq C t^{-2} \int_{M} H^{2}\left(x, y, \frac{t}{2}\right) d y .
$$

Since $\epsilon_{0}>0$ is arbitrary, this shows that $\Delta H(x, \cdot, t) \in L^{2}(M)$. In particular, the function $H(x, \cdot, t) \Delta H(x, \cdot, t)$ is integrable.

Let $\eta_{\epsilon}$ be a non-negative cut-off function defined on $M$ with support on $M_{\epsilon}$. Choose $\eta_{\epsilon}$ such that it satisfies $\eta_{\epsilon}=1$ on $M_{2 \epsilon}$ and $\left|\nabla \eta_{\epsilon}\right| \leq \frac{5}{\epsilon}$. For any $x \in M \backslash \Sigma_{M}$, let us consider

$$
\begin{aligned}
\int_{M} \eta_{\epsilon}^{2}(y) H(x, y, t) \Delta H(x, y, t) d y \\
=-\int_{M} \eta_{\epsilon}^{2}(y)|\nabla H|^{2}(x, y, t) d y \\
\quad-2 \int_{M} \eta_{\epsilon}(y) H(x, y, t)\left\langle\nabla \eta_{\epsilon}(y), \nabla H(x, y, t)\right\rangle d y .
\end{aligned}
$$


Using the fact that the support of $\nabla \eta_{\epsilon}$ is on $M_{\epsilon, 2 \epsilon}=M_{\epsilon} \backslash M_{2 \epsilon}$, the second term can be estimated by

$$
\begin{aligned}
& 2\left|\int_{M} \eta_{\epsilon}(y) H(x, y, t)\left\langle\nabla \eta_{\epsilon}(y), \nabla H(x, y, t)\right\rangle d y\right| \\
& \quad \leq 2 \int_{M_{\epsilon, 2 \epsilon}}\left|\nabla \eta_{\epsilon}\right|^{2}(y) H^{2}(x, y, t) d y+\frac{1}{2} \int_{M_{\epsilon, 2 \epsilon}} \eta_{\epsilon}^{2}(y)|\nabla H|^{2}(x, y, t) d y .
\end{aligned}
$$

Using the estimate $\left|\nabla \eta_{\epsilon}\right| \leq \frac{5}{\epsilon}$ and the fact that $H(x, y, t)$ is bounded in $M$, the first term on the right-hand side is dominated by

$$
2 \int_{M_{\epsilon, 2 \epsilon}}\left|\nabla \eta_{\epsilon}\right|^{2}(y) H^{2}(x, y, t) d y \leq C(t) \epsilon^{-2} V(\epsilon, 2 \epsilon)
$$

where $V(\epsilon, 2 \epsilon)$ is the volume of $M_{\epsilon, 2 \epsilon}$ and $C(t)=\sup _{x, y \in M} \tilde{H}\left(r_{x}(y), t,\right)$. The fact that $\Sigma_{M}$ is of at least real codimension 2 implies that

$$
2 \int_{M_{\epsilon, 2 \epsilon}}\left|\nabla \eta_{\epsilon}\right|^{2}(y) H^{2}(x, y, t) d y \leq C_{1}(t)
$$

for some constant $C_{1}(t)>0$ which depends only on $t$ and $M$. Combining this with (3.1), we have

$$
\begin{aligned}
\frac{1}{2} \int_{M}|\nabla H|^{2}(x, y, t) d y & \leq-\int_{M} H(x, y, t) \Delta H(x, y, t) d y+C_{1}(t) \\
& =\frac{-1}{2}\left(\frac{\partial}{\partial t} \int_{M} H^{2}(x, y, t) d y\right)+C_{1}(t) \\
& =\frac{-1}{2}\left(\frac{\partial}{\partial t} H(x, x, 2 t)\right)+C_{1}(t) .
\end{aligned}
$$

To estimate the time derivative, we use the local gradient estimate of [L-Y] which asserts that

$$
|\nabla H|^{2}(x, x, s)-H(x, x, s) \frac{\partial H}{\partial s}(x, x, s) \leq C_{2} H^{2}(x, x, s)
$$

where $C_{2}>0$ depends on $s, M$, and the distance from $x$ to $\Sigma_{M}$. Letting $s=2 t$, this implies that

$$
\frac{-1}{2}\left(\frac{\partial}{\partial t} H(x, x, 2 t)\right) \leq C_{2} H(x, x, 2 t),
$$

and hence the function $H(x, \cdot, t) \in H_{1,2}(M)$.

Using this fact, we claim that

$$
\int_{M} H(x, y, t) \Delta H(z, y, t) d y=-\int_{M}\langle\nabla H(x, y, t), \nabla H(z, y, t)\rangle d y
$$

for all $x, z \in M \backslash \Sigma_{M}$. Indeed, as in (3.1) we have

$$
\begin{aligned}
\int_{M} \eta_{\epsilon}^{2}(y) H(x, y, t) \Delta H(z, y, t) d y \\
=-\int_{M} \eta_{\epsilon}^{2}(y)\langle\nabla H(x, y, t), \nabla H(z, y, t)\rangle d y \\
\quad-2 \int_{M} \eta_{\epsilon}(y) H(x, y, t)\left\langle\nabla \eta_{\epsilon}(y), \nabla H(z, y, t)\right\rangle d y .
\end{aligned}
$$


The second term on the right-hand side can be estimated by

$$
\begin{aligned}
2 \mid \int_{M} & \eta_{\epsilon}(y) H(x, y, t)\left\langle\nabla \eta_{\epsilon}(y), \nabla H(z, y, t)\right\rangle d y \mid \\
& \leq 2 C(t) \int_{M}\left|\nabla \eta_{\epsilon}(y)\right||\nabla H|(z, y, t) d y \\
& \leq 2 C(t)\left(\int_{M_{\epsilon, 2 \epsilon}}\left|\nabla \eta_{\epsilon}\right|^{2}(y) d y\right)^{\frac{1}{2}}\left(\int_{M_{\epsilon, 2 \epsilon}}|\nabla H|^{2}(z, y, t) d y\right)^{\frac{1}{2}} .
\end{aligned}
$$

Observe that the term $\int_{M_{\epsilon, 2 \epsilon}}\left|\nabla \eta_{\epsilon}\right|^{2}(y) d y$ is bounded when $\epsilon \rightarrow 0$ as argued before, while the second term $\int_{M_{\epsilon, 2 \epsilon}}|\nabla H|^{2}(z, y, t) d y \rightarrow 0$ because $H(z, \cdot, t) \epsilon$ $H_{1,2}(M)$. The claim now follows by letting $\epsilon \rightarrow 0$ in (3.3).

To see that $\int_{M} H(x, y, t) d y=1$, we consider

$$
\begin{aligned}
\frac{\partial}{\partial t} \int_{M} \eta_{\epsilon}(y) H(x, y, t) d y & =\int_{M} \eta_{\epsilon}(y) \Delta H(x, y, t) d y \\
& =-\int_{M}\left\langle\nabla \eta_{\epsilon}(y), \nabla H(x, y, t)\right\rangle d y
\end{aligned}
$$

where $\eta_{\epsilon}$ is the cut-off function defined above. Applying the Schwarz inequality, we see that for $0<t_{1}<t_{2}$ we have

$$
\begin{aligned}
& \left|\int_{M} \eta_{\epsilon}(y) H\left(x, y, t_{2}\right) d y-\int_{M} \eta_{\epsilon}(y) H\left(x, y, t_{1}\right) d y\right| \\
& \quad \leq \int_{t_{1}}^{t_{2}}\left|\frac{\partial}{\partial t} \int_{M} \eta_{\epsilon}(y) H(x, y, t) d y\right| d t \\
& \quad \leq \int_{t_{1}}^{t_{2}}\left(\int_{M_{\epsilon, 2 \epsilon}}\left|\nabla \eta_{\epsilon}\right|^{2}(y) d y\right)^{\frac{1}{2}}\left(\int_{M_{\epsilon, 2 \epsilon}}|\nabla H|^{2}(x, y, t) d y\right)^{\frac{1}{2}} d t .
\end{aligned}
$$

The right-hand side tends to 0 as $\epsilon \rightarrow 0$, hence

$$
\int_{M} H\left(x, y, t_{2}\right) d y=\int_{M} H\left(x, y, t_{1}\right) d y .
$$

Since $\lim _{t_{1} \rightarrow 0} \int_{M} H\left(x, y, t_{1}\right) d y=1$, it follows that the conservation property $\int_{M} H(x, y, t) d y=1$ is valid for all $x \in M \backslash \Sigma_{M}$.

Lemma 3.2. Let $K_{\epsilon}(x, y, t)$ be the Neumann heat kernel defined on the manifold $M_{\epsilon}$. Then $K_{\epsilon}(x, y, t)$ converges to $H(x, y, t)$ on compact subsets of $\left(M \backslash \Sigma_{M}\right) \times\left(M \backslash \Sigma_{M}\right) \times(0, \infty)$.

Proof. By the local estimate of [L-Y], we conclude that $K_{\epsilon}(x, y, t)$ is uniformly bounded on compact subsets of $M_{\epsilon} \times M_{\epsilon}$. Hence together with the gradient estimate, $K_{\epsilon}$ converges to some kernel $K$ on compact subsets of $\left(M \backslash \Sigma_{M}\right) \times$ $\left(M \backslash \Sigma_{M}\right) \times(0, \infty)$. Note that the maximum principle implies that $H \leq K$. 
Moreover, for any $x \in M \backslash \Sigma_{M}$ and for $0<\epsilon<\epsilon^{\prime}$, we have

$$
\begin{aligned}
\int_{M_{\epsilon^{\prime}}} H(x, y, t) d y & \leq \int_{M_{\epsilon^{\prime}}} K(x, y, t) d y \\
& =\lim _{\epsilon \rightarrow 0} \int_{M_{\epsilon^{\prime}}} K_{\epsilon}(x, y, t) d y \\
& \leq \lim _{\epsilon \rightarrow 0} \int_{M_{\epsilon}} K_{\epsilon}(x, y, t) d y \\
& =1 .
\end{aligned}
$$

Letting $\epsilon \rightarrow 0$ and using the fact that $\int_{M} H(x, y, t) d y=1$, we conclude that $H \equiv K$.

Lemma 3.3. The time derivatives of the heat kernel $\frac{\partial^{k} H}{\partial t^{k}}(x, y, t)$ are in $H_{1,2}(M)$. In fact, there exist constants $C_{3}(k), C_{4}(k)>0$ depending only on $k$, such that

$$
\int_{M}\left|\frac{\partial^{k} H}{\partial t^{k}}\right|^{2}(x, y, t) d y \leq C_{3}(k) t^{-2 k} \int_{M} H^{2}\left(x, y, \frac{t}{2}\right) d y
$$

and

$$
\int_{M}\left|\nabla \frac{\partial^{k} H}{\partial t^{k}}\right|^{2}(x, y, t) d y \leq C_{4}(k) t^{-2 k-1} \int_{M} H^{2}\left(x, y, \frac{t}{2}\right) d y .
$$

Proof. This follows by combining the argument of Lemma 3.1 and Lemma 7 of [C-L-Y1].

\section{SELF-ADJOINTNESS OF THE LAPLACIAN ON A SINGULAR VARIETY}

In this section, we will discuss the issue of self-adjointness of the Laplacian on an algebraic variety with respect to the induced Fubini-Study metric from $\mathbb{C P}^{n+l}$. Let us consider the domain $\mathscr{D}(d)$ of the operator $d$ to be the set of all $C^{1}$ functions $f$ on $M \backslash \Sigma_{M}$ which is in $L^{2}$ and whose exterior derivative is also in $L^{2}$. Similarly, we also define the domain $\mathscr{D}(\delta)$ of the operator $\delta$ to be the set of $C^{1}$ 1-forms $\omega$ on $M \backslash \Sigma_{M}$ which is in $L^{2}$ and $\delta \omega$ is also in $L^{2}$. The domain of the Laplacian $\mathscr{D}(\Delta)$ is then defined to be the set of $C^{2}$ functions on $M \backslash \Sigma_{M}$ such that $f \in \mathscr{D}(d)$ and $d f \in \mathscr{D}(\delta)$. In [G], Gaffney showed that if $d$ and $\delta$ are adjoint to each other with respect to their domains, then the closure $\bar{\Delta}$ of the Laplacian $\Delta=-\delta d$ is self-adjoint.

Theorem 4.1. The closure $\bar{\Delta}$ of the Laplacian defined on functions is self-adjoint on a compact (not necessarily complex) variety $M$ (without boundary) whose singular set $\Sigma_{M}$ is at least of real codimension 2 .

Proof. In view of Gaffney's theorem, in order to show that $\bar{\Delta}$ is self-adjoint, we only need to show that

$$
(d f, \omega)=(f, \delta \omega)
$$

for $f \in \mathscr{D}(d)$ and $\omega \in \mathscr{D}(\delta)$. If we know that any function in $H_{1,2}(M)$ can be approximated by functions in $H_{1,2}^{c}\left(M_{\epsilon}\right)=\left\{f \in H_{1,2}\left(M_{\epsilon}\right) \mid f=0\right.$ on $\left.\partial M_{\epsilon}\right\}$ 
where $M_{\epsilon}$ is the complement of the $\epsilon$-tubular neighborhood of $M$, then (4.1) follows quite easily. To see this, we first observe that the domain of $d$ is a subset of $H_{1,2}(M)$. If we can find a sequence $f_{i} \in H_{1,2}^{c}\left(M_{\epsilon_{i}}\right)$ such that $f_{i} \rightarrow f \in H_{1,2}(M)$, then

$$
\begin{aligned}
(d f, \omega) & =\lim _{i \rightarrow \infty}\left(d f_{i}, \omega\right) \\
& =\lim _{i \rightarrow \infty}\left(f_{i}, \delta \omega\right) \\
& =(f, \delta \omega)
\end{aligned}
$$

where integration by parts is justified because $f_{i}$ satisfies the Dirichlet boundary condition.

Let us now consider the question of whether functions in $H_{1,2}(M)$ can be approximated by functions in $H_{1,2}^{c}\left(M_{\epsilon}\right)$. First of all, we would like to show that the set of functions in $H_{1,2}(M)$ which are bounded is dense in $H_{1,2}(M)$. For any $f \in H_{1,2}(M)$, let us define the function $g_{a}$ by

$$
g_{a}(x)= \begin{cases}f(x), & \text { if }|f(x)| \leq a, \\ a, & \text { if } f(x)>a, \\ -a, & \text { if } f(x)<-a .\end{cases}
$$

Let us also define the set $N_{a}=\{x \in M|| f(x) \mid>a\}$. Clearly

$$
\begin{aligned}
a^{2} V\left(N_{a}\right) & \leq \int_{N_{a}} f^{2} \\
& \leq \int_{M} f^{2} .
\end{aligned}
$$

Hence $V\left(N_{a}\right) \leq a^{-2} \int_{M} f^{2}$ which tends to 0 as $a \rightarrow \infty$. Since $f \in H_{1,2}(M)$, this implies that $\int_{N_{a}} f^{2}$ and $\int_{N_{a}}|\nabla f|^{2}$ both tend to 0 as $a \rightarrow \infty$. Therefore

$$
\begin{aligned}
\int_{M}\left|f-g_{a}\right|^{2} & =\int_{N_{a}}(|f|-a)^{2} \\
& \leq \int_{N_{a}}|f|^{2} \rightarrow 0
\end{aligned}
$$

and

$$
\int_{M}\left|\nabla\left(f-g_{a}\right)\right|^{2}=\int_{N_{a}}|\nabla f|^{2} \rightarrow 0 .
$$

This justifies the denseness of bounded functions in $H_{1,2}(M)$.

Let us now assume that $f \cdot \in H_{1,2}(M)$ is bounded. Define $f_{\epsilon}=\phi_{\epsilon} f \in$ $H_{1,2}^{c}\left(M_{\epsilon}\right)$ where $\phi_{\epsilon}$ is a cut-off function with support in $M_{\epsilon^{\prime}}$ for $\epsilon^{\prime}=$ $\frac{1}{2} \exp \left(-\epsilon^{-2}\right)$, and $\phi_{\epsilon}=1$ on $M_{\epsilon}$. Clearly the $L^{2}$-norm of $f-f_{\epsilon}$ tends to 0 as $\epsilon \rightarrow 0$. Let us consider

$$
\int_{M}\left|\nabla\left(f-f_{\epsilon}\right)\right|^{2}=\int_{M_{\epsilon^{\prime}, \epsilon}}\left|\nabla\left(f-f_{\epsilon}\right)\right|^{2}+\int_{T_{\epsilon^{\prime}}}|\nabla f|^{2}
$$


where $M_{\epsilon^{\prime}, \epsilon}=M_{\epsilon} \backslash M_{\epsilon^{\prime}}$ and $T_{\epsilon^{\prime}}$ is the $\epsilon^{\prime}$-tubular neighborhood of the singular set. Clearly

$$
\int_{T_{\epsilon^{\prime}}}|\nabla f|^{2} \rightarrow 0
$$

as $\epsilon^{\prime} \rightarrow 0$ because $f \in H_{1,2}(M)$, and

$$
\begin{aligned}
\int_{M_{\epsilon^{\prime}, \epsilon}}\left|\nabla\left(f-f_{\epsilon}\right)\right|^{2} & =\int_{M_{\epsilon^{\prime}, \epsilon}}|\nabla f|^{2}-2 \int_{M_{\epsilon^{\prime}, \epsilon}}\left\langle\nabla f, \nabla f_{\epsilon}\right\rangle+\int_{M_{\epsilon^{\prime}, \epsilon}}\left|\nabla f_{\epsilon}\right|^{2} \\
& \leq 2 \int_{M_{\epsilon^{\prime}, \epsilon}}|\nabla f|^{2}+2 \int_{M_{\epsilon^{\prime}, \epsilon}}\left|\nabla f_{\epsilon}\right|^{2} .
\end{aligned}
$$

Observe that the first term on the right-hand side of (4.2) tends to 0 , hence we only need to consider the second term. Using the definition of $f_{\epsilon}$ we have

$$
\begin{aligned}
\int_{M_{\epsilon^{\prime}, \epsilon}}\left|\nabla f_{\epsilon}\right|^{2} & =\int_{M_{\epsilon^{\prime}, \epsilon}} f^{2}\left|\nabla \phi_{\epsilon}\right|^{2}+2 \int_{M_{\epsilon^{\prime}, \epsilon}} f \phi_{\epsilon}\left\langle\nabla \phi_{\epsilon}, \nabla f\right\rangle+\int_{M_{\epsilon^{\prime}, \epsilon}} \phi_{\epsilon}^{2}|\nabla f|^{2} \\
& \leq 2 \int_{M_{\epsilon^{\prime}, \epsilon}} f^{2}\left|\nabla \phi_{\epsilon}\right|^{2}+2 \int_{M_{\epsilon^{\prime}, \epsilon}} \phi_{\epsilon}^{2}|\nabla f|^{2} .
\end{aligned}
$$

The term $\int_{M_{\epsilon^{\prime}},} \phi_{\epsilon}^{2}|\nabla f|^{2} \rightarrow 0$, and the issue is reduced to showing that the first term $\int_{M_{\epsilon^{\prime}, \epsilon}} f^{2}\left|\nabla \phi_{\epsilon}\right|^{2}$ also tends to. 0 . By the assumption that $f$ is bounded, it suffices to show that

$$
\int_{M_{\epsilon^{\prime}, \epsilon}}\left|\nabla \phi_{\epsilon}\right|^{2} \rightarrow 0
$$

Now, as suggested in [C-F], we choose $\phi_{\epsilon}$ to be

$$
\phi_{\epsilon}= \begin{cases}\left(\frac{r}{\epsilon}\right)^{\epsilon}, & 2 \epsilon^{\prime} \leq r \leq \epsilon, \\ \left(\frac{2 \epsilon^{\prime}}{\epsilon}\right)^{\epsilon}\left(\frac{r}{\epsilon^{\prime}}-1\right), & \epsilon^{\prime} \leq r \leq 2 \epsilon^{\prime}, \\ 0, & 0 \leq r \leq \epsilon^{\prime}\end{cases}
$$

where $r$ denotes the distance function to $\Sigma_{M}$. Clearly,

$$
\left|\nabla \phi_{\epsilon}\right|^{2} \leq \begin{cases}\left(\frac{r}{\epsilon}\right)^{2 \epsilon-2}, & 2 \epsilon^{\prime} \leq r \leq \epsilon, \\ \left(\frac{2 \epsilon^{\prime}}{\epsilon}\right)^{2 \epsilon}\left(\frac{1}{\left(\epsilon^{\prime}\right)^{2}}\right), & \epsilon^{\prime} \leq r \leq 2 \epsilon^{\prime} \\ 0, & 0 \leq r \leq \epsilon^{\prime} .\end{cases}
$$

Using the fact that the singular set is at most of real codimension 2 , the area of 
$\partial T_{r}$ is at most linear in $r$ for sufficiently small $r$. Hence we can estimate

$$
\begin{aligned}
\int_{M_{\epsilon^{\prime}, \epsilon}}\left|\nabla \phi_{\epsilon}\right|^{2} & \leq C \int_{2 \epsilon^{\prime}}^{\epsilon}\left(\frac{r}{\epsilon}\right)^{2 \epsilon-2} r d r+C \int_{\epsilon^{\prime}}^{2 \epsilon^{\prime}}\left(\frac{2 \epsilon^{\prime}}{\epsilon}\right)^{2 \epsilon}\left(\frac{r}{\left(\epsilon^{\prime}\right)^{2}}\right) d r \\
& \left.\left.\leq C \frac{r^{2 \epsilon}}{2 \epsilon^{2 \epsilon-1}}\right]_{2 \epsilon^{\prime}}^{\epsilon}+C\left(\frac{2 \epsilon^{\prime}}{\epsilon}\right)^{2 \epsilon}\left(\frac{r^{2}}{2\left(\epsilon^{\prime}\right)^{2}}\right)\right]_{\epsilon^{\prime}}^{2 \epsilon^{\prime}} \\
& =C\left(\frac{\epsilon}{2}+\frac{2^{2 \epsilon-1} \epsilon^{\prime}}{\epsilon^{2 \epsilon-1}}+\frac{3}{2}\left(\frac{2 \epsilon^{\prime}}{\epsilon}\right)^{2 \epsilon}\right) \\
& =C\left(\frac{\epsilon}{2}+\frac{2^{2 \epsilon-2} \exp \left(-\epsilon^{-2}\right)}{\epsilon^{2 \epsilon-1}}+\frac{3}{2}\left(\frac{\exp \left(-2 \epsilon^{-1}\right)}{\epsilon^{2 \epsilon}}\right)\right) .
\end{aligned}
$$

Obviously, the right-hand side tends to 0 as $\epsilon \rightarrow 0$. This establishes the selfadjointness of $\bar{\Delta}$.

\section{SOME APPLiCATIONS}

Using the argument in $\S 4$, we will first establish that the operator $(\Delta-1)^{-1}$ is compact on $L^{2}(M)$ and hence the spectrum of $\Delta$ is discrete and given by a set of eigenvalues

$$
\left\{0=\lambda_{0}<\lambda_{1} \leq \lambda_{2} \leq \cdots \leq \lambda_{k} \leq \cdots \rightarrow \infty\right\} .
$$

To see this, let us first show that the heat operator is contractive in $L^{2}(M)$.

Lemma 5.1. Let the heat operator $e^{\Delta t}$ be defined by

$$
e^{\Delta t} f(x)=\int_{M} H(x, y, t) f(y) d y
$$

for any $f \in L^{2}(M)$. Then $e^{\Delta t}$ is contractive in $L^{2}(M)$.

Proof. Let $f \in L^{2}(M)$. By the fact that $H(x, \cdot, t) \in L^{2}(M)$, the heat operator maps $L^{2}(M)$ to $L^{2}(M)$. Differentiating the square of the $L^{2}$-norm with respect to $t$ and applying (3.2), we have

$$
\begin{aligned}
\frac{\partial}{\partial t}\left\|e^{\Delta t} f\right\|_{L^{2}}^{2} & =2 \int_{M}\left(\Delta e^{\Delta t} f\right) e^{\Delta t} f \\
& =2 \int_{M} \int_{M} \int_{M} H(x, y, t) \Delta H(z, y, t) d y f(x) f(z) d x d z \\
& =-2 \int_{M} \int_{M} \int_{M}\langle\nabla H(x, y, t), \nabla H(z, y, t)\rangle d y f(x) f(z) d x d z \\
& =-2 \int_{M}\left|\nabla e^{\Delta t} f\right|^{2} d y \\
& \leq 0 .
\end{aligned}
$$

This shows that $e^{\Delta t}$ is contractive in $L^{2}$. 
Lemma 5.2. The operator $(\Delta-1)^{-1}: L^{2}(M) \rightarrow H_{1,2}(M)$ is bounded.

Proof. Clear $\Delta-1$ is invertible and its inverse $(\Delta-1)^{-1}$ is given by the kernel

$$
(\Delta-1)^{-1} f(x)=\int_{M} \int_{0}^{\infty} e^{-t} H(x, y, t) f(y) d y .
$$

By Lemma 5.1, we have

$$
\begin{aligned}
\|(\Delta & -1)^{-1} f \|_{L^{2}}^{2} \\
& =\int_{0}^{\infty} \int_{0}^{\infty} \int_{M \times M \times M} e^{-(s+t)} H(x, y, t) H(z, y, s) f(x) f(z) d y d x d z d t d s \\
& =\int_{0}^{\infty} \int_{0}^{\infty} \int_{M \times M} e^{-(s+t)} H(x, z, t+s) f(x) f(z) d x d z d t d s \\
& =\int_{0}^{\infty} \int_{0}^{\infty} \int_{M \times M \times M} e^{-(s+t)} H\left(x, y, \frac{s+t}{2}\right) \\
& =\int_{0}^{\infty} \int_{0}^{\infty} \int_{M} e^{-(s+t)}\left|e^{\Delta \frac{s+t}{2}} f\right|^{2} \\
& \leq\|f\|_{L^{2}}^{2} \int_{0}^{\infty} \int_{0}^{\infty} e^{-(s+t)} d s d t \\
& \leq\|f\|_{L^{2}}^{2} .
\end{aligned}
$$

This shows that if $h=(\Delta-1)^{-1} f$, then $\|h\|_{L^{2}} \leq\|f\|_{L^{2}}$. Also, because

$$
\begin{aligned}
\|f\|_{L^{2}}^{2} & =\|(\Delta-1) h\|_{L^{2}}^{2} \\
& =\|\Delta h\|_{L^{2}}^{2}-2 \int_{M} h \Delta h+\|h\|_{L^{2}}^{2}
\end{aligned}
$$

and the Schwarz inequality

$$
\left|2 \int_{M} h \Delta h\right| \leq \frac{1}{2}\|\Delta h\|_{L^{2}}^{2}+2\|h\|_{L^{2}}^{2},
$$

we have

$$
\begin{aligned}
\|f\|_{L^{2}}^{2} & \geq \frac{1}{2}\|\Delta h\|_{L^{2}}^{2}-\|h\|_{L^{2}}^{2} \\
& \geq \frac{1}{2}\|\Delta h\|_{L^{2}}^{2}-\|f\|_{L^{2}}^{2}
\end{aligned}
$$

This implies that

$$
\|\Delta h\|_{L^{2}} \leq 2\|f\|_{L^{2}}
$$

and

$$
\begin{aligned}
\|d h\|_{L^{2}}^{2} & \leq\|\Delta h\|_{L^{2}}\|h\|_{L^{2}} \\
& \leq 2\|f\|_{L^{2}}^{2}
\end{aligned}
$$

which proves the lemma. 
Theorem 5.3. Let $M$ be an algebraic variety in $\mathbb{C P}^{n+l}$. The operator $(\Delta-1)^{-1}$ is a compact operator in $L^{2}(M)$. In particular, $\Delta$ has discrete spectrum.

Proof. In view of Lemma 5.2, it suffices to show that the inclusion map $H_{1,2}(M)$ $\subset L^{2}(M)$ is compact. To see this, let $\left\{f_{i}\right\}$ be a bounded sequence in $H_{1,2}(M)$ with

$$
\left\|f_{i}\right\|_{L^{2}(M)}^{2}+\left\|\nabla f_{i}\right\|_{L^{2}(M)}^{2} \leq A .
$$

Let $\epsilon_{k} \rightarrow 0$ be a positive sequence of sufficiently small numbers and $\epsilon_{k}^{\prime}=$ $\frac{1}{2} \exp \left(-\epsilon_{k}^{-2}\right)$. For each $k$ and $i$, let us define the function $f_{i}^{k}=\phi_{\epsilon_{k}} f_{i}$ where $\phi_{\epsilon_{k}}$ is the cut-off function used in $\S 4$. One checks easily that $\left\{f_{i}^{k}\right\}_{i=1}^{\infty}$ is a bounded sequence in $H_{1,2}^{c}\left(M_{\epsilon_{k}^{\prime}}\right)$. By the compactness of the inclusion map $H_{1,2}^{c}\left(M_{\epsilon_{k}^{\prime}}\right) \subset$ $L^{2}\left(M_{\epsilon_{k}^{\prime}}\right)$ there is a subsequence of $\left\{f_{i}^{1}\right\}$ denoted by $\left\{f_{1_{i}}^{1}\right\}$ which converges to a function $f^{1} \in L^{2}\left(M_{\epsilon_{1}^{\prime}}\right)$. Inductively, we can find a subsequence $\left\{f_{k_{i}}^{k}\right\}$ of the sequence $\left\{f_{(k-1)_{i}}^{k}\right\}$ which converges to some function $f^{k} \in L^{2}\left(M_{\epsilon_{k}^{\prime}}\right)$. We now claim that the sequence $\left\{f^{k}\right\}$ converges to a function $f \in L^{2}(M)$ as $\epsilon \rightarrow 0$ and that $f$ is in $L^{2}(M)$.

By the definition of $\phi_{\epsilon_{k}}, f_{j}^{k}=f_{j}$ on $M_{\epsilon_{k}}$. Hence for $k>j$,

$$
\left\|f^{k}-f^{j}\right\|_{L^{2}} \leq\left\|f^{k}-f_{k_{i}}^{k}\right\|_{L^{2}}+\left\|f_{k_{i}}^{k}-f_{k_{i}}^{j}\right\|_{L^{2}}+\left\|f_{k_{i}}^{j}-f^{j}\right\|_{L^{2}} .
$$

Since the sequence $\left\{f_{k_{i}}^{k}\right\}$ is a subsequence of $\left\{f_{j_{i}}^{j}\right\}$, for $i$ sufficiently large we may assume that

$$
\left\|f^{k}-f_{k_{i}}^{k}\right\|_{L^{2}} \leq \frac{1}{k}
$$

and

$$
\left\|f_{k_{i}}^{j}-f^{j}\right\|_{L^{2}} \leq \frac{1}{k}
$$

The term $\left\|f_{k_{i}}^{k}-f_{k_{i}}^{j}\right\|_{L^{2}}$ can be estimated by

$$
\begin{aligned}
\left\|f_{k_{i}}^{k}-f_{k_{i}}^{j}\right\|_{L^{2}}^{2} & =\int_{T_{\epsilon_{j}}}\left(\phi_{\epsilon_{k}}-\phi_{\epsilon_{j}}\right)^{2} f_{k_{i}}^{2} \\
& \leq \int_{T_{\epsilon_{j}}} f_{k_{i}}^{2} .
\end{aligned}
$$

Let us observe that since an algebraic variety is a minimal current in $\mathbb{C P}^{n+l}$, after choosing a fixed embedding of $\mathbb{C P}^{n+l}$ into some Euclidean space, the variety $M$ can be viewed as a current in Euclidean space with bounded mean curvature. The Sobolev inequality of Michael-Simon [M-S] (see also [S]) asserts that there exists a constant $C>0$ depending only on $M$ such that for any function $g \in H_{1,2}(M)$, we have

$$
\left(\int_{M}|g|^{\frac{2 n}{n-1}}\right)^{\frac{n-1}{n}} \leq C\left(\int_{M}|\nabla g|^{2}+\int_{M}|g|^{2}\right) .
$$


On the other hand, applying the Scharwz inequality yields

$$
\begin{aligned}
V\left(T_{\epsilon_{j}}\right)^{-\left(\frac{1}{n}\right)} \int_{T_{\epsilon_{j}}}|g|^{2} & \leq\left(\int_{T_{\epsilon_{j}}}|g|^{\frac{2 n}{n-1}}\right)^{\frac{n-1}{n}} \\
& \leq\left(\int_{M}|g|^{\frac{2 n}{n-1}}\right)^{\frac{n-1}{n}} .
\end{aligned}
$$

Hence combining with (5.5) and setting $g=f_{k_{i}}$ we have

$$
\int_{T_{\epsilon_{j}}}\left|f_{k_{i}}\right|^{2} \leq A C V\left(T_{\epsilon_{j}}\right)^{\frac{1}{n}}
$$

Since $V\left(T_{\epsilon_{j}}\right) \rightarrow 0$ as $j \rightarrow 0$, we conclude that

$$
\int_{T_{\epsilon_{j}}}\left|f_{k_{i}}\right|^{2} \rightarrow 0
$$

uniformly for the sequence $\left\{f_{k_{i}}\right\}$ as $j \rightarrow 0$. Therefore combining with (5.1), (5.2), (5.3), and (5.4), this shows that the sequence $\left\{f^{j}\right\}$ is a Cauchy sequence, hence converges to some function $f \in L^{2}(M)$.

Clearly $f$ is an accumulation point of the original sequence $\left\{f_{i}\right\}$. To see this, given any $\epsilon>0$, we can find a $k_{0}$ such that for all $k \geq k_{0}$ we have

$$
\left\|f-f^{k}\right\|_{L^{2}} \leq \epsilon .
$$

We can also find a sufficiently large $i$ such that

$$
\left\|f^{k}-f_{k_{i}}^{k}\right\|_{L^{2}} \leq \epsilon \text {. }
$$

On the other hand,

$$
\begin{aligned}
\left\|f_{k_{i}}^{k}-f_{k_{i}}\right\|_{L^{2}}^{2} & \leq \int_{T_{\epsilon_{k}}}\left|f_{k_{i}}\right|^{2} \\
& \leq A C V\left(T_{\epsilon_{k}}\right)^{\frac{1}{n}} .
\end{aligned}
$$

Choosing $k$ sufficiently large, and using (5.6) and (5.7), we conclude that

$$
\left\|f-f_{k_{i}}\right\|_{L^{2}} \leq 3 \epsilon \text {. }
$$

This proves the theorem.

Corollary 5.4. Let $M^{n}$ be an $n$-dimensional algebraic variety. The heat operator $e^{\Delta t}$ is of trace class with

$$
\begin{aligned}
\operatorname{Tr} e^{\Delta t} & =\int_{M} H(x, x, t) d x \\
& \leq V(M) \tilde{H}(0, t) .
\end{aligned}
$$

In particular, the pointwise trace of the heat kernel must satisfy the estimate

$$
H(x, x, t) \leq(4 \pi t)^{-n}\left(1+\frac{s(n)}{6} t+O\left(t^{2}\right)\right)
$$


as $t \rightarrow 0$, where $s(n)=4 n(n+1)$ is the scalar curvature of $\mathbb{C P}^{n}$. In particular, the zeta function satisfies

$$
\sum_{i=0}^{\infty} e^{-\lambda_{i} t} \leq(4 \pi t)^{-n}\left(V(M)\left(1+\frac{s(n)}{6} t\right)+O\left(t^{2}\right)\right)
$$

and the eigenvalues satisfy the asymptotic estimate

$$
\lambda_{i} \geq\left(\frac{(2 \pi)^{2 n} i}{\omega_{2 n} V(M)}\right)^{\frac{1}{n}}
$$

as $i \rightarrow \infty$ where $\omega_{2 n}$ is the volume of the unit $2 n$-ball in $\mathbb{R}^{2 n}$.

Proof. From the construction of $H$, the estimate of Theorem 2.1 holds for $H$. The corollary now follows from the asymptotic behavior of $\tilde{H}(0, t)$ (see [B-G-M]).

Corollary 5.5. Let $M^{n}$ be an algebraic variety of degree $d$. There exists a constant $C(n, d)>0$ depending only on $n$ and $d$ alone, such that the $d^{\text {th }}$ non-zero eigenvalue with respect to the Bergmann metric on $M$ satisfies

$$
\lambda_{d} \geq C(n, d) \text {. }
$$

Proof. The estimate of Theorem 2.1 asserts that

$$
H(x, x, t) \leq \tilde{H}(0, t)
$$

where $\tilde{H}(0, t)$ is the pointwise trace of the heat kernel on $\mathbb{C P}^{n}$. Integrating over $M$ and using the fact that $\tilde{H}(0, t)$ is independent on $x$, we have

$$
\begin{aligned}
1+\sum_{i=1}^{\infty} e^{-\lambda_{i} t} & \leq V(M) \tilde{H}(0, t) \\
& =\frac{V(M)}{V\left(\mathbb{C} \mathbb{P}^{n}\right)}\left(1+\sum_{i=1}^{\infty} e^{-\tilde{\lambda}_{i} t}\right) \\
& \leq \frac{V(M)}{V\left(\mathbb{C} \mathbb{P}^{n}\right)}\left(1+C(n) t^{-n}\right)
\end{aligned}
$$

where $\tilde{\lambda}_{i}$ is the $i^{\text {th }}$ non-zero eigenvalue of $\mathbb{C P}^{n}$ and $C(n)>0$ is a constant depending only on $n$. Using the fact that $V(M)=d V\left(\mathbb{C P}^{n}\right)$, we have

$$
1-d+\sum_{i=1}^{\infty} e^{-\lambda_{i} t} \leq d C(n) t^{-n}
$$

Let us now substitute $t=\frac{\alpha}{\lambda_{d}}$ and obtain

$$
1-d+d e^{-\alpha} \leq d C(n)\left(\frac{\lambda_{d}}{\alpha}\right)^{n} .
$$

This implies that

$$
\lambda_{d} \geq \alpha\left(\frac{1-d\left(1-e^{-\alpha}\right)}{d C(n)}\right)^{\frac{1}{n}}
$$


It is clear that if we choose $\alpha$ sufficiently small so that $1-d\left(1-e^{-\alpha}\right)>0$, then

$$
\lambda_{d} \geq C(n, d)
$$

for some constant depending only on $n$ and $d$.

Let us remark that it is impossible to get a similar uniform lower bound for the lower eigenvalues. This can be seen by considering a sequence of subvarieties that tends to $d$ copies of $\mathbb{C P}^{n}$. In this case, the first $d-1$ non-zero eigenvalues will tend to 0 . It is interesting to view the estimate of Corollary 5.5 as an analogy to the lower estimate of Schoen-Wolpert-Yau [S-W-Y] for the $(2 g-2)^{\frac{t h}{h}}$ nonzero eigenvalue on a compact Riemann surface with genus $g$.

\section{REFERENCES}

[B-G-M] M. Berger, P. Gauduchon, and E. Mazet, Le spectre d'une variété riemannienne, Lecture Notes in Math., vol. 194, Springer, New York, 1974.

[C-F] 1. Chavel and E. Feldman, Spectra of domains in compact manifolds, J. Funct. Anal. 30 (1978), 198-222.

[C-Y] J. Cheeger and S. T. Yau, A lower bound for the heat kernel, Comm. Pure Appl. Math. 34 (1981), 465-480.

[C-L-Y1] S. Y. Cheng, P. Li, and S. T. Yau, On the upper estimate of the heat kernel of a complete Riemannian manifold, Amer. J. Math. 103 (1981), 1021-1063.

[C-L-Y2] __, Heat equations on minimal submanifolds and their applications, Amer. J. Math. 106 (1984), 1033-1065.

[G] M. Gaffney, The harmonic operator for exterior differential forms, Proc. Nat. Acad. Sci. U.S.A. 37 (1951), 48-50.

[L] P. Li, Eigenvalue estimates on homogeneous manifolds, Comment. Math. Helv. 55 (1980), 347-363.

[L-Y] P. Li and S. T. Yau, On the parabolic kernel of the Schrödinger operator, Acta Math. 156 (1986), 153-201.

[M-S] J. H. Michael and L. Simon, Sobolev and mean-value inequalities on generalized submanifolds of $\mathbb{R}^{n}$, Comm. Pure Appl. Math. 26 (1973), 361-379.

[N] M. Nagase, On the heat operators of normal singular algebraic surfaces, J. Differential Geometry 28 (1988), 37-57.

[P1] V. Pati, The heat trace on singular algebraic threefolds, J. Differential Geometry 37 (1993), 245-261.

[P2] _ The Laplacian on threefolds with isolated singularities, preprint.

[S-W-Y] R. Schoen, S. Wolpert, and S. T. Yau, Geometric bounds on the low eigenvalues of a compact surface, Geometry of the Laplace Operator, Proc. Sympos. Pure Math., vol. 36, Amer. Math. Soc., Providence, RI, 1980, pp. 279-285.

[S] L. Simon, Lectures on geometric measure theory, Proc. Centre for Mathematical Analysis, Australian National Univ., Canberra, 1984.

Department of Mathematics, University of California, Irvine, California 92717-3875

E-mail address: pli@math.uci.edu

Courant Institute, New York University, New York, New York 10012

E-mail address: tiang@taotao.cims.nyu.edu 